\title{
Caracterización de la comunidad de malezas y su diversidad en una modelación estadística en un cultivo de duraznero (Prunus persica (L.) Batsch.)
}

\section{Characterization of the weed community and its diversity in a statistical modeling in peach (Prunus persica (L.) Batsch.) orchard}

\author{
Oscar Eduardo Moreno-Preciado'; Helber Enrique Balaguera-López²
}

\begin{abstract}
${ }^{1}$ Ing. Agrónomo, Facultad de Ciencias Agropecuarias. Universidad Pedagógica y Tecnológica de Colombia. Tunja - Boyacá, Colombia; e-mail: oscar. morenopreciado@uptc.edu.co; (D) https://orcid.org/0000-0001-8796-6307

${ }^{2}$ Ing. Agrónomo, M.Sc., Ph.D., Profesor asociado Universidad Nacional de Colombia, Facultad de Ciencias Agrarias, Departamento de Agronomía. Bogotá, Colombia; e-mail: hebalagueral@unal.edu.co; (1D https://orcid.org/0000-0003-3133-0355
\end{abstract}

Cómo citar: Moreno-Preciado, O.E.; Balaguera-López, H.E. 2021. Caracterización de la comunidad de malezas y su diversidad en una modelación estadística en un cultivo de duraznero (Prunus persica (L.) Batsch.). Rev. U.D.C.A Act. \& Div. Cient. 24(1):e1734. http://doi.org/10.31910/rudca.v24.n1.2021.1734

Artículo de acceso abierto publicado por Revista U.D.C.A Actualidad \& Divulgación Científica, bajo una Licencia Creative Commons CC BY-NC 4.0

Publicación oficial de la Universidad de Ciencias Aplicadas y Ambientales U.D.C.A, Institución de Educación Superior Acreditada de Alta Calidad por el Ministerio de Educación Nacional.

Recibido: octubre 12 de $2020 \quad$ Aceptado: enero 21 de $2021 \quad$ Editado por: Ingeborg Zenner de Polanía

\section{RESUMEN}

En los frutales, incluido el duraznero, las malezas son uno de los principales problemas que afectan la productividad y la calidad y se considera necesario conocer aspectos de la biología y la ecología de estas especies, para realizar un correcto manejo integral. El objetivo de este estudio fue realizar la caracterización de las especies de malezas presentes en el cultivo de duraznero (Prunus persica (L.) Batsch), determinando los índices de diversidad y el uso de un modelo geoespacial, para observar su distribución en campo. Se seleccionó un cultivo comercial de 4,5ha de duraznero variedad Robidoux, se muestrearon 15 puntos aleatoriamente, mediante un cuadro de $0,25 \mathrm{~m}^{2}$; con las especies de malezas presentes en cada cuadro, se determinaron los índices de valor de importancia (IVI), de dominancia (1-D), Shannon-Wiener ( $\mathrm{H}^{\prime}$ ) y Pielou (E). A estos índices, se les realizó modelación de la variabilidad espacial, mediante interpolación polinomial local. La representatividad relativa con los estimadores Chao 2 y Jacknife 1 confirmó que se documentó el 79 y $72 \%$ de las especies presentes, respectivamente. Se identificaron 12 especies, de las cuales, Oxalis corniculata, Modiola caroliniana, Lolium temulentum y Fuertesimalva limensis presentaron el mayor IVI y estas especies, se consideran como las de mayor interferencia en el cultivo. Se encontró que la diversidad fue baja, no es equitativa y presenta dominancia de $O$. corniculata y $M$. caroliniana. La modelación geoespacial de los índices de diversidad reportó una variación importante en todo el cultivo. Esta información, se convierte en una herramienta útil para el manejo integral de malezas en el cultivo de duraznero.

Palabras clave: Competencia; Arvenses; Shannon-Wiener; Caducifolio. 


\section{ABSTRACT}

In fruit trees, including peach trees, weeds are one of the main problems that affect productivity and quality, and it is considered necessary to know aspects of the biology and ecology of these species to carry out a correct integral management. The objective of the study was to characterize the weed species present in the peach orchard (Prunus persica (L.) Batsch), by determining the diversity indices and using a geospatial model of their distribution in the field. A commercial Robidoux variety of 4.5 ha peach orchard was selected, where 15 randomly distributed points were sampled through a $0.25 \mathrm{~m}^{2}$ square, with the weed species present in each square, the index of importance value (IVI), dominance index (1-D), Shannon-Wiener $\left(\mathrm{H}^{\prime}\right)$ and Pielou (E) were determined. These indices were modeled for spatial variability through local polynomial interpolation. The relative representativeness with the Chao 2 and Jacknife 1 estimators confirmed that $79 \%$ and $72 \%$ of the weed species were documented, respectively. Twelve species were identified, of which Oxalis corniculata, Modiola caroliniana, Lolium temulentum, and Fuertesimalva limensis presented the highest IVI, and these species are considered as the ones with the greatest interference in crop. The diversity was found to be low, not equitable, and dominated by $O$. corniculata and $M$. caroliniana. The geospatial modeling of the diversity indices reported an important variation throughout the crop. This information becomes a useful tool for integral management of weeds in the peach orchards.

Keywords: Competition; Weeds; Shannon-Wiener; Deciduou.

\section{INTRODUCCIÓN}

En Colombia, el duraznero [Prunus persica (L.) Batsch, familia Rosaceae], es el cultivo caducifolio de mayor importancia (Pinzón et al. 2014), representando, para el 2018, el 31\% (2.501,62ha) del área cultivada y el 31\% (34.738,09t) también de la producción del total de los caducifolios producidos en el país (Agronet, 2018). Boyacá es el departamento con mayor producción (Patiño \& Miranda, 2013), registrando un valor de 14.255,86t, en 925,60ha (Agronet, 2018). En el país, el duraznero se cultiva en zonas con temperaturas promedio de 13 a $19^{\circ} \mathrm{C}$, entre los 1.800 y $2.800 \mathrm{~m}$ s.n.m., regímenes de lluvia monomodales y bimodales y brillo solar promedio de 1.400 horas/ año (Pinzón et al. 2014).

Actualmente, el cultivo de duraznero presenta grandes retos, que están relacionados con el cambio climático, tecnologías pre y poscosecha, con la adaptación a diferentes mercados y la necesidad de reducir el uso de insumos (Fachinello et al. 2011; Patiño \& Miranda, 2013), incluidos los herbicidas, que en un plan manejo integrado de malezas deben ser la última opción, respecto a métodos alternativos de control (culturales, mecánicos, biotecnólogico y biológico) (Marquez, 2019). Se conoce que, en el manejo del duraznero, el 88,3\% de los productores colombianos realizan control de malezas, del cual, el 25,5\% lo hace únicamente de forma manual o mecánica (corte con guadaña); el 35,7\%, de forma química con herbicidas a base de glifosato y paraquat y un $38,8 \%$ de los productores combinan los controles manuales o mecánicos con el control químico (Ávila et al. 2013).

Para disminuir el efecto negativo de las malezas y para hacer un uso más eficiente de los herbicidas de síntesis química, con menor impacto ambiental y lograr mayor rentabilidad del sistema de producción, se requiere el uso del manejo integral de malezas (MIM). Este manejo es entendido como un sistema de toma de decisiones para la selección y uso de las prácticas de control simples o armónicamente coordinadas de estrategias de manejo; se basa en el costo-beneficio del impacto producido, social y medio ambientalmente (Zimdahl, 2018). El MIM, se debe basar, de forma primordial, en el conocimiento de la biología y la ecología de las malezas dentro del cultivo, tal como lo reportan Ramírez et al. (2015) y Quintero-Pertuz et al. (2020); sin embargo, en el cultivo de duraznero del trópico alto colombiano, se desconoce gran parte de estos aspectos fundamentales.

De acuerdo con Storkey \& Neve (2018), realizar estudios de caracterización de la comunidad de malezas es importante, porque brinda información para el manejo en el cultivo. Estos mismos autores mencionan que una comunidad de malezas más diversa será menos competitiva, por tanto, la diversidad taxonómica de las malezas, se ha propuesto como un indicador de la sostenibilidad general de un cultivo (Storkey \& Neve, 2018). De esta manera, los inventarios florísticos de malezas permiten comparar la diversidad entre dos o más comunidades de diferentes hábitats, o un mismo hábitat a lo largo del tiempo, o gradientes ambientales naturales y antrópicos (Campo \& Duval, 2014). Las malezas, se encuentran como agrupaciones de varias especies, de las cuales, algunas son más competitivas que otras (Zimdahl, 2018). Su clasificación permite identificar las familias y las especies más limitantes en los cultivos, conocimiento que facilita crear estrategias para determinar el momento en que son más vulnerables y aplicar el manejo más eficiente (Cobb \& Reade, 2010).

Para realizar el plan de manejo integrado, se debe usar, como herramienta, los índices de diversidad. Los más usados en malherbología son: el índice de valor de importancia (IVI), que permiten tener una visión, tanto de la composición como de la distribución de las especies de plantas en una comunidad dada, al asignar una categoría a cada especie con relación a su importancia relativa (Tauseef et al. 2012; Cardenal et al. 2016). También están los índices diversidad, que combinan la evaluación de dos aspectos separados importantes, que son la riqueza e igualdad (Nagendra, 2002); los más comúnmente usados son: el índice de equidad de Pielou (E), de Shannon-Wiener $\left(\mathrm{H}^{\prime}\right)$ y el índice de dominancia Simpson (D) (Juárez et al. 2007). Estos últimos son medidas que se basan en la composición florística y evalúan qué tan homogénea o biodiversa es una comunidad (Cardenal et al. 2016). Igualmente, se incluye la curva de acumulación de especies (CAE), método, con el cual, se determina la cantidad total de especies presentes en el estudio, a medida que se agregan más unidades de muestreo adicionales al conjunto de muestras reunidas anteriormente (Rodríguez, 2013) y, de esta forma, calcular el número de muestreo faltantes, para obtener un muestreo fiable. También es útil modelar 
estos índices geoespacialmente, para generar una recomendación de manejo mucho más precisa y objetiva. $\mathrm{Al}$ respecto, varios estudios mencionan la importancia de este tipo de metodologías en el MIM (Calha et al. 2014; Somervill et al. 2020).

En Colombia, se han realizado estudios importantes de reconocimiento de malezas asociados a cultivos de arroz (Fuentes et al. 2006; Ramírez et al. 2015), espinaca (Rodríguez et al. 2008), banano (Quintero Pertuz et al. 2020), cítricos, guayaba, maracuyá y piña (Hoyos et al. 2015), entre otros, y en frutales de clima frío, como la uchuva (Plaza \& Pedraza, 2007); sin embargo, en el cultivo de duraznero, no existe este tipo de reportes.

Por lo anterior, el estudio tuvo como objetivo realizar la caracterización de las especies de malezas presentes en el cultivo de duraznero, determinando los índices de valor de importancia, dominancia Simpson, Shannon-Wiener, equidad Pielou y el uso de un modelo geoespacial, para observar su distribución en campo.

\section{MATERIALES Y MÉTODOS}

El estudio, se realizó entre julio y septiembre del 2019, en un cultivo comercial de duraznero (P. persica L.) variedad Rubidoux, de 4,5ha, perteneciente a la granja experimental "Tunguavita", de la Universidad Tecnológica y Pedagógica de Colombia (UPTC), municipio de Paipa (Boyacá - Colombia), ubicada a latitud de $5^{\circ}$ $45^{\prime}$ Norte y longitud $73^{\circ} 45^{\prime}$ Oeste y a una altitud de $2.525 \mathrm{~m}$ s.n.m. La zona presenta un régimen pluvial bimodal, con un promedio de $881 \mathrm{~mm} /$ año y temperatura media de $14^{\circ} \mathrm{C}$, con máximos de 20 $22^{\circ} \mathrm{C}$ y mínimos en época seca, de menos de $5^{\circ} \mathrm{C}$, humedad relativa promedio de 70\%, en época seca y 78\%, de lluvias (IDEAM, 2012).

El muestreo, se realizó usando una imagen satelital DEM reciente (abril-2019) del cultivo, del satélite ALOS, por su resolución espacial de $15 \mathrm{~m}$. Luego, se hizo una rejilla de $0,25 \mathrm{~m}^{2}$, a partir de la cual, se seleccionaron 15 puntos distribuidos aleatoriamente, como se muestra en la figura 1, siguiendo las recomendaciones de Jamaica \& Plaza (2014). Las coordenadas, se importaron a un GPS, en un archivo KML, para realizar la ubicación de cada punto en campo; posteriormente, en cada punto, se lanzó al azar un marco de PVC de $0,25 \mathrm{~m}^{2}$, teniendo la precaución de que el cuadro no cayera sobre los árboles de duraznero. Con los resultados del muestreo, se calcularon los estimadores de riqueza y se realizó la curva de acumulación de especie, para verificar la calidad del muestreo y calcular los muestreos extra necesarios, para conseguir un catálogo fiable, el cual, se confirmó usando un método estadístico no paramétrico, conocido como estimadores de riqueza, específicamente, los estimadores Chao2 y Jacknife1 (Gonzales et al. 2010).

Por cada punto muestreado, se identificaron las especies de malezas presentes, usando las guías de Gámez et al. (2018) y Fuentes et al. (2011). También, se determinó el número de individuos por especies (abundancia) y se hizo un registro fotográfico de cada punto; posteriormente, en el software Photoshop, se corrigió la perspectiva de la imagen; con la herramienta "medición”, se calculó, por medio de polígonos, el área de cobertura de cada especie identificada, tomando como referencia, un lado del recuadro de muestreo, que mide $50 \mathrm{~cm}$.

Con los datos de abundancia por punto de muestreo, se determinaron los estimadores de riqueza Chao2 y Jacknife1 y se suavizaron los valores de acumulación de especies por número de muestras, para graficar la curva de acumulación de especies (CUE), mediante el programa Estimates 9.1. Con los estimadores de riqueza calculados, se estimó la representatividad relativa $(\mathrm{Rr})$ del catálogo, de acuerdo con Magurran (2004):

$$
R r=\frac{\text { Valor de estimador }}{S} * 100
$$

Donde, $\mathrm{S}=$ total de especies encontradas en el muestreo

El índice de valor de importancia (IVI) fue calculado con los parámetros de densidad absoluta (Da), densidad relativa (Dr), frecuencia absoluta $(\mathrm{Fa})$, frecuencia relativa $(\mathrm{Fr})$, cobertura absoluta $(\mathrm{Ca})$, cobertura relativa $(\mathrm{Cr})$ por especie, de acuerdo con Prates et al. (2019) y Ramírez et al. (2015).

$$
\begin{aligned}
I V I & =\text { Dr }+F r+C r \\
D a & =\frac{\text { Número de individuos por especie }}{\text { número de muestras totales }} \\
D r & =\frac{\text { densidad absoluta por especie }}{\text { densidad total de todas las especies }} \\
F a & =\frac{\text { Frecuencia por especie }}{\text { número total de sitios }} \\
F r & =\frac{\text { Frecuencia absoluta por especie }}{\text { Frecuencia total de todas las especies }} \\
C a & =\frac{\text { Área ocupada por especie }}{\text { Área total de muestreo }} \\
C r & =\frac{\text { cobertura absoluta por especie }}{\text { absoluta de todas las especies }}
\end{aligned}
$$

Los índices de diversidad Alfa $(\alpha)$ : índice dominancia Simpson (D), índice Shannon-Wiener $\left(\mathrm{H}^{\prime}\right)$ e índice de equidad Pielou, se calcularon con las siguientes fórmulas (Rodríguez, 2013):

$$
\begin{aligned}
& D-\sum\left(\frac{n_{i}}{N}\right)^{2} \\
& H^{\prime}=-\sum\left[p_{i}\left(\ln p_{i}\right)\right] \\
& E=\frac{H^{\prime}}{\operatorname{Ln} S}
\end{aligned}
$$




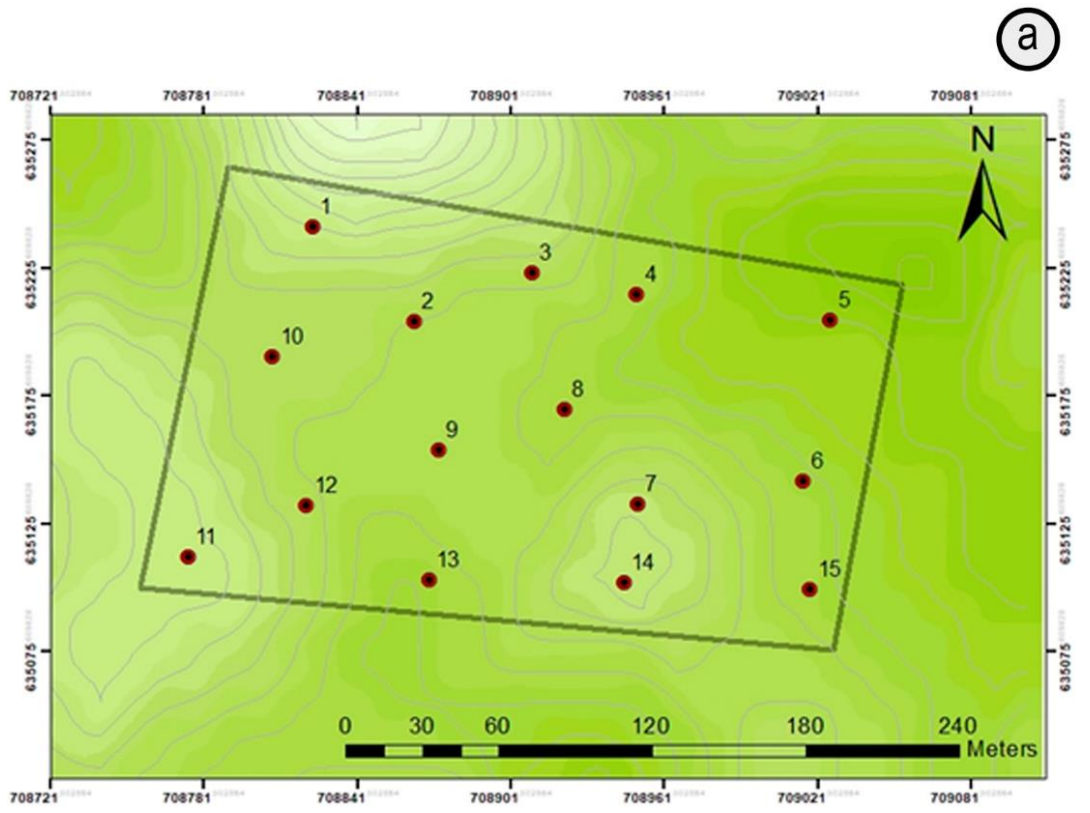

(b)

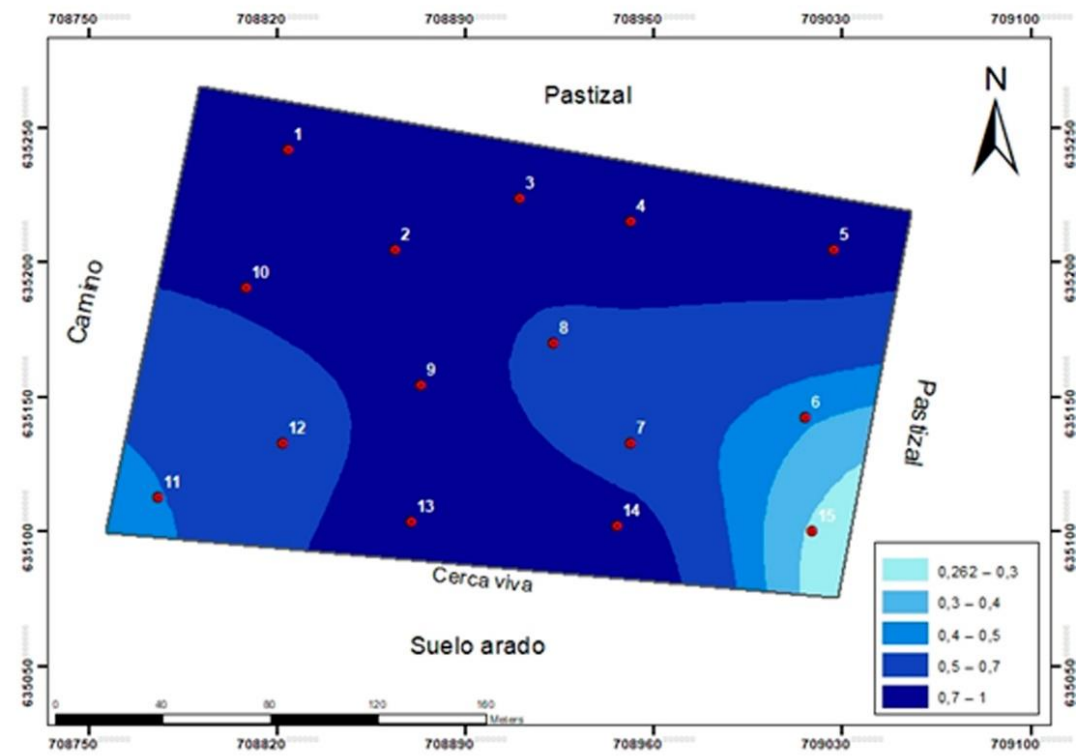

Figura 1. a. distribución geoespacial de puntos de muestreo en la zona de estudio; b. modelación por interpolación polinomial local de la diversidad dominancia, donde: 0 representa alta dominancia y 1 baja dominancia.

Donde, $n_{i}=$ número de individuos de las especies i, $\mathrm{N}=$ número total de individuos de todas las especies, $\mathrm{P}_{\mathrm{i}}=$ abundancia proporcional de una especie dada, $\mathrm{E}=$ medida de uniformidad Pielou, $\mathrm{H}=$ diversidad Shannon-Wiener calculada, $\mathrm{S}=$ número total de especies.

Para observar el comportamiento geoespacial de los índices de diversidad, se modelaron en ArcMap10,8, usando los valores individuales de los 15 puntos de muestreo obtenidos en el software Past3, con el método de interpolación polinomial local. Este método, se basa en un diagrama de dispersión, en la que la verdadera función presenta uno o más picos o valles (mínimos o máximos relativos), dando una aproximación satisfactoria a la función de regresión (Devore, 2012). En los mapas obtenidos, se asignaron tonalidades oscuras a las zonas de baja diversidad y claras, a las de alta diversidad.

\section{RESULTADOS Y DISCUSIÓN}

La representatividad relativa con los estimadores Chao2 y Jacknife 1 confirmó que se documentó el 79 y $72 \%$ de las especies presentes, respectivamente. Según Magurran (2004), una representatividad por encima del $70 \%$ es válida para hacer inferencia de comunidades, donde las especies faltantes se consideran raras o de poca abundancia, 
confirmando que no es requerido realizar más muestreos. Las especies estimadas fueron 15, para Chao 2 y 17, para Jacknife 1. Cuando se hizo la CAE basados en estos mismos estimadores, se obtuvo que es suficiente con 10 o 13 muestreos para Chao2 y Jacknife1, respectivamente, para lograr un catálogo fiable (Figura 2), mientras que para un catálogo completo habrían sido necesarios 22 y 28 muestreos, respectivamente.
Se identificaron 12 especies pertenecientes a 8 familias y 12 géneros, agrupados en 10 dicotiledóneas y 2 monocotiledóneas, denotando la predominancia de especies dicotiledóneas (Tabla 1). Es importante indicar que 9 especies son de ciclo anual $(75 \%)$ y 3 de ciclo perenne (25\%), siendo Oxalis corniculata L. Small, la especie que presentó mayor densidad, con 1.358 individuos (68\%), seguida por Modiola caroliniana, 226 (11\%) y Fuertesimalva limensis, 186 (9\%) (Tabla 1). De

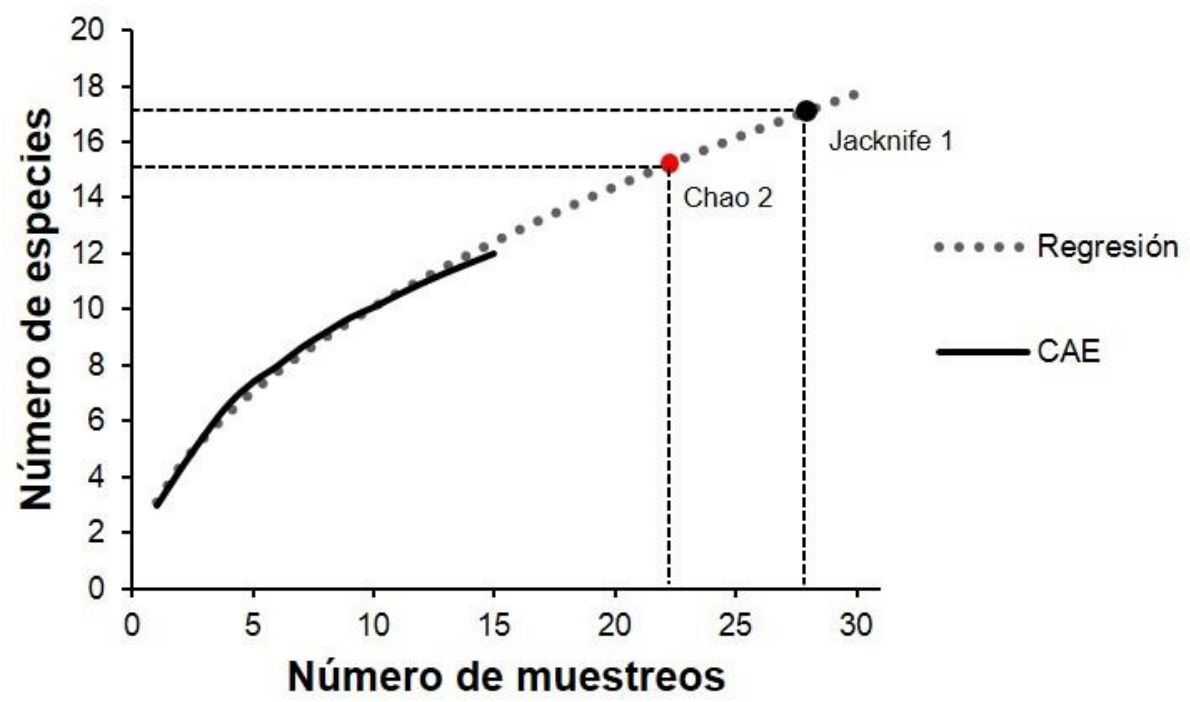

Figura 2. Curva de acumulación de especies (CAE): proyección número de muestreos vs riqueza estimada Chao 2 y Jacknife 1.

las 8 familias identificadas todas son consideradas de importancia económica, principalmente, porque afectan el rendimiento de los cultivos (Duke, 2018).

La familia Asteraceae (3 géneros/3 especies) presentó la mayor cantidad de especies, seguida por la familia Malvaceae $(2 / 2)$ y Poaceae (2/2); estas tres familias abarcaron el 58\% del total de las especies encontradas (Tabla 1). Al respecto, Plaza \& Pedraza (2007), en cultivos de uchuva de Boyacá y Cundinamarca, también reportan a estas familias.

Las especies más frecuentes fueron O. corniculata $(33 \%)$, seguida de $L$. temulentum $(16 \%)$ y $S$. oleraceus $(12 \%)$; estas tres especies presentaron el $61 \%$ de la cobertura total. O. corniculata también mostró la mayor cobertura y dominancia, seguida de $M$. carolinata (Tabla 2). En $B$. rapa, se observó baja densidad $(0,7 \%)$ y frecuencia $(2,3 \%)$, pero una cobertura de $9 \%$, denotando su alta competencia por el espacio (Zimdahl, 2018).

El análisis del IVI indicó que solo 4 especies abarcaron el 80\% del índice: O. corniculata (44\%), seguido por M. caroliniana (17\%), L. temulentum $(10 \%)$ y F. limensis (9\%), encontrándose que, a excepción de $L$. temulentum, que presenta ciclo anual, O. corniculata es perenne (Tibuhwa, 2016) y M. caroliniana y F. limensis son anuales-perenne
(Gámez et al. 2018); estas especies pueden ser las que tendrían más habilidad para causar daño mediante interferencia (Concenço et al. 2019). El índice de dominancia Simpson (D) fue de 0,511, lo que indica una dominancia media. Este resultado difiere de Vera et al. (2018), quienes encontraron valores de 0,93, en Musáceas, valor que indica alta dominancia de especies. Cardenal et al. (2016) reportan índices de 0,27 a 1 en el banco activo de semillas, en un cultivo de zanahoria. Además, calculando el inverso del índice 1/D (1,96), se determinó que dos especies presentaron dominancia en el cultivo, de acuerdo con el valor IVI (Tabla 2). Estas especies fueron $O$. corniculata (valor IVI de 0,44), especie hospedera de diversas plagas y enfermedades que pueden afectar al cultivo, como Botrytis sp. (Castro et al. 2019) y $M$. caroliniana $(0,17)$, que pese a ser hospedera de enfermedades (Albu et al. 2019; Aime \& Abbasi, 2018), no hay reporte de que afecte al cultivo de duraznero; sin embargo, M. caroliniana puede causar dificultad en labores culturales, hay posibilidad de que afecte el sistema de fertirriego, principalmente, porque puede crecer en la zona de los emisores y es una planta muy ramificada, que presenta tallos lignificados (Fuentes et al. 2011). Además, se dificulta su control en etapas avanzadas de desarrollo.

El índice Pielou, valor 0,460, determinó que la comunidad no es equitativa, es poco diversa, acumulando el 94\% de los individuos colectados, en solo 4 especies: $O$. corniculata (1.358 individuos, que 
Tabla 1. Clasificación de especies encontradas en el muestreo en el cultivo de duraznero. Subclase D: Dicotiledónea, M: Monocotiledonea; Ciclo A: Anual, P: Perenne.

\begin{tabular}{|c|c|c|c|c|}
\hline Subclase & Familia & Especie & Nombre común & Ciclo \\
\hline \multirow{10}{*}{$\mathrm{D}$} & \multirow{3}{*}{ Asteraceae } & Senecio vulgaris L. & Cineraria, Yuyito, Senecio & A \\
\hline & & Sonchus oleraceus L. & Cerraja, Canayuyo, Lechosa & A \\
\hline & & Taraxacum officinale F.H. Wigg & Diente de león & $\mathrm{P}$ \\
\hline & \multirow{2}{*}{ Malvaceae } & Fuertesimalva limensis (L.) Fryxell & Malva blanca & $\mathrm{A}-\mathrm{P}$ \\
\hline & & Modiola caroliniana (L.) G. Don & Malva, Malva tendida & $A-P$ \\
\hline & Brassicaceae & Brassica rapa $\mathrm{L}$. & Nabo amarillo & A \\
\hline & Caryophyllaceae & Spergula arvensis L. & Cilantrillo & A \\
\hline & Oxalidaceae & Oxalis corniculata (L.) Small & Trebol agrio & $\mathrm{P}$ \\
\hline & Plantaginaceae & Veronica persica Poir & $\begin{array}{l}\text { Veronica, Azulita, } \\
\text { Golondrina }\end{array}$ & A \\
\hline & Poligonaceae & Polygonium seguetum Kunth & Barbasco, Gualola & $\mathrm{P}$ \\
\hline \multirow{2}{*}{ M } & \multirow{2}{*}{ Poaceae } & Lolium temulentum $\mathrm{L}$. & Vallico, Ray grass & A \\
\hline & & Poa annua $\mathrm{L}$ & Pasto gallina & $A$ \\
\hline
\end{tabular}

Tabla 2. Índice de Valor de Importación (IVI). Do: Dominancia, Fr: frecuencia, Co: Cobertura de las especies de malezas encontradas en el cultivo de duraznero.

\begin{tabular}{|l|c|c|c|c|c|c|c|}
\hline \multirow{2}{*}{ Especies } & \multicolumn{3}{|c|}{ Valor absoluto } & \multicolumn{3}{c|}{ Valor relativo } & \multirow{2}{*}{$\begin{array}{c}\text { IVI } \\
\text { (\%) }\end{array}$} \\
\cline { 2 - 8 } & Do & Fr & Co & Do & Fr & Co & (xalis corniculata \\
\hline Modiola caroliniana & 1358 & 0,93 & 0,17 & 0,68 & 0,33 & 0,32 & 44 \\
\hline Lolium temulentum & 226 & 0,27 & 0,15 & 0,11 & 0,09 & 0,29 & 17 \\
\hline Fuertesimalva limensis & 106 & 0,47 & 0,04 & 0,05 & 0,16 & 0,08 & 10 \\
\hline Sonchus oleraceus & 186 & 0,27 & 0,04 & 0,09 & 0,09 & 0,07 & 9 \\
\hline Brassica rapa & 18 & 0,33 & 0,05 & 0,01 & 0,12 & 0,09 & 7 \\
\hline Poligonium seguetum & 13 & 0,07 & 0,05 & 0,01 & 0,02 & 0,09 & 4 \\
\hline Senecio vulgaris & 5 & 0,07 & 0,03 & 0,00 & 0,02 & 0,05 & 2 \\
\hline Spergula arvensis & 4 & 0,13 & 0,01 & 0,00 & 0,05 & 0,01 & 2 \\
\hline Taraxacum officinale & 62 & 0,07 & 0,00 & 0,03 & 0,02 & 0,00 & 2 \\
\hline Veronica persica & 5 & 0,13 & 0,00 & 0,00 & 0,05 & 0,00 & 2 \\
\hline Poa annua & 11 & 0,07 & 0,00 & 0,01 & 0,02 & 0,00 & 1 \\
\hline
\end{tabular}

representan el 68\% de la muestra), M. caroliniana (226), F. limensis (186) y L. tumulentum (106). El índice Shannon-Wiener $(1,14)$ muestra una baja diversidad, denotando la presencia de pocas especies y, en algunas de ellas, un alto número de individuos, como en el caso de O. corniculata (Tabla 3); por el contrario, se reportan índices mayores, que van desde 2,3 a 2,7 en las comunidades de malezas en cultivos de arroz (Ramírez et al. 2015) y en Musáceas, con un índice de 3,22 (Vera et al. 2018) e indican que son comunidades más diversas y que presentan más especies por área.
Se evidencia en los índices hallados en el cultivo de duraznero que presenta una baja diversidad y dominancia media, permitiendo la implementación adecuada de métodos de control no químicos, como el mecánico, cultural, preventivo, alelopático o biológico (Jabran \& Chauhan, 2018), basado en la biología de las especies limitantes.

El modelo de los índices permitió detallar la distribución geoespacial de las características de la diversidad (dominancia, equidad de individuos y abundancia de especies). El modelo de dominancia 
toma valores de 0-1 representados en tonalidades claras a oscuras (Figura 1B), donde el valor de 0 representa cuando hay tantas especies diferentes como individuos y 1 , cuando todos los individuos pertenecen a la misma especie (Rodríguez, 2013). Este modelo mostró la presencia de una amplia zona, donde se presenta dominancia de una sola especie (azul oscuro). Con base en el IVI se puede determinar que las dos especies más dominantes son: $O$. corniculata y $M$. carolinata, se pueden hallar en esta zona. En las zonas con menor dominancia (azul claro), se muestra un claro efecto de borde hacia la zona donde el suelo esta desprovisto de cobertura vegetal; sin embargo, las especies presentes en estos puntos con menor dominancia (punto 11, 15 y 6) (Tabla 3), se identificaron las especies O. corniculata, L. temulentum, B. rapa, F. limensis, T. offiniale, que pueden llegar a ser limitantes agronómicamente, siendo de especial interés la caracterización de estos puntos, para iniciar un plan control preventivo.

La modelación de Shannon-Wiener (Figura 3A) mostró cómo el número de especies presentes por área disminuye en dirección surnorte. Este, comportamiento, también se observó en el modelo de la equidad (Figura 3B), donde el número de individuos por especies van siendo desiguales a medida que se distancian de los puntos de muestreo 11 y 15, los cuales, presentaron la mayor diversidad en distribución y abundancia de especies. Las zonas donde se encuentran estos puntos se pueden determinar como importantes entradas para el banco de semilla del cultivo que, pese a presentar una mayor diversidad, en este caso, dada por especies que pueden presentar una amenaza, especialmente, en suelos poco perturbados (Zimdahl, 2018), como es el caso de los frutales, se puede deber, a la abundancia de especies que se desarrollan en estos suelos trabajados, que aumentan la oferta de semillas que pueden ser transportadas por el viento, agua o mecánicamente por el hombre, maquinaria y herramientas o animales (Duke, 2018); por esta razón, es importante el manejo de estas zonas para controlar su propagación y permitir el desarrollo de especies de interés agronómico.

El estudio mostró que de doce especies identificadas (88\% dicotiledóneas y $12 \%$ monocotiledóneas) (Tabla 1), dos presentan dominancia en el cultivo $O$. corniculata y $M$. carolinata, encontrándose la distribución de la dominancia en la mayor parte del cultivo, al igual de dos puntos muy diversos en los límites con suelos sin cobertura (Figura 3), que se pueden presentar como entradas de semillas de malezas al cultivo.

El cálculo de un solo valor de cualquier índice de diversidad para un lote, resulta poco objetivo, para generar una estrategia de manejo eficiente de las malezas, debido a que las especies consideradas como malezas tienen diferentes formas de distribución en el lote, como la distribución regular, al azar y en parches, propio de su comportamiento ecológico (Zimdahl, 2018); por esta razón, la distribución geoespacial de los índices de diversidad, se convierte en una herramienta más robusta y completa, como base para un manejo integral de malezas en los sistemas agrícolas, principalmente, si se quiere realizar un manejo por sitio especifico, de esta manera, se puede hacer un uso más eficiente de los herbicidas (Calha $e t$ al. 2014). Somervill et al. (2020) también plantean la importancia y los retos del uso de mapas y modelos espaciales de la distribución de poblaciones de malezas, como base para el manejo integral de estas especies.

Tabla 3. Distribución de abundancia de especies de malezas por punto de muestreo en el cultivo de duraznero.

\begin{tabular}{|c|c|c|c|c|c|c|c|c|c|c|c|c|c|c|c|}
\hline \multirow{2}{*}{ Especie } & \multicolumn{15}{|c|}{ Número de especies por punto } \\
\hline & 1 & 2 & 3 & 4 & 5 & 6 & 7 & 8 & 9 & 10 & 11 & 12 & 13 & 14 & 15 \\
\hline Oxalis corniculata & 761 & 7 & 2 & 78 & 20 & 8 & 79 & 61 & 2 & 302 & 17 & 12 & 1 & 0 & 8 \\
\hline Senecio vulgaris & 3 & 0 & 0 & 0 & 1 & 0 & 0 & 0 & 0 & 0 & 0 & 0 & 0 & 0 & 0 \\
\hline Sonchus oleraceus & 2 & 0 & 1 & 1 & 0 & 0 & 12 & 0 & 0 & 0 & 0 & 2 & 0 & 0 & 0 \\
\hline Lolium temulentum & 0 & 0 & 14 & 0 & 1 & 8 & 39 & 33 & 0 & 0 & 3 & 0 & 0 & 0 & 8 \\
\hline Poa annua & 0 & 0 & 0 & 1 & 0 & 0 & 0 & 0 & 0 & 0 & 0 & 0 & 0 & 0 & 0 \\
\hline Modiola caroliniana & 0 & 123 & 0 & 0 & 0 & 0 & 0 & 0 & 0 & 0 & 35 & 0 & 59 & 0 & 9 \\
\hline Fuertesimalva limensis & 0 & 2 & 0 & 0 & 0 & 2 & 0 & 0 & 18 & 0 & 0 & 0 & 0 & 164 & 0 \\
\hline Taraxacum officinale & 0 & 0 & 0 & 0 & 0 & 1 & 4 & 0 & 0 & 0 & 0 & 0 & 0 & 0 & 0 \\
\hline Veronica persica & 0 & 0 & 0 & 0 & 0 & 0 & 11 & 0 & 0 & 0 & 0 & 0 & 0 & 0 & 0 \\
\hline Spergula arvensis & 0 & 0 & 0 & 0 & 0 & 0 & 0 & 0 & 0 & 62 & 0 & 0 & 0 & 0 & 0 \\
\hline Brassica rapa & 0 & 0 & 0 & 0 & 0 & 0 & 0 & 0 & 0 & 0 & 0 & 0 & 0 & 0 & 13 \\
\hline Poligonium seguetum & 0 & 0 & 0 & 0 & 0 & 0 & 0 & 0 & 0 & 0 & 0 & 5 & 0 & 0 & 0 \\
\hline
\end{tabular}



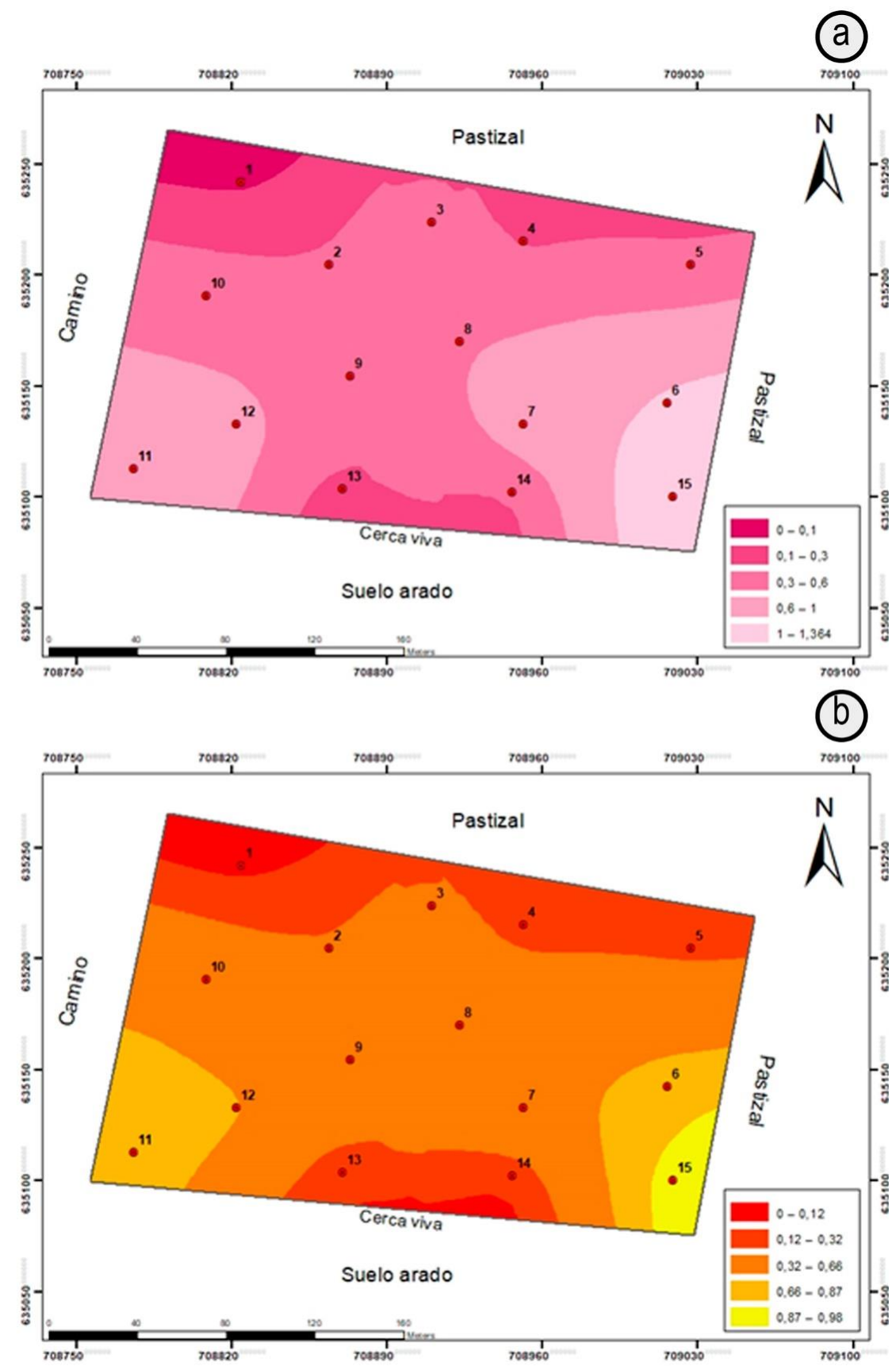

Figura 3. Modelación por interpolación polinomial local del a. índice Shannon-Wiener, donde: el número de especies aumenta a medida que los valores son mayores a cero $(0)$ y b. del índice de equidad Pielou, donde: 0 representa distribución de los individuos por especie inequitativa y 1 distribución equitativa. Los números 1 al 15 corresponden a los sitios de muestreo.

Agradecimientos: A la Ingeniera Agrónoma Lula Marina Sánchez, por su valioso apoyo para la realización del trabajo en la fase de campo. Conflicto de intereses: El manuscrito fue preparado y revisado con la participación de todos los autores, quienes declaramos que no existe conflicto de intereses que ponga en riesgo la validez de los resultados presentados.

\section{REFERENCIAS}

1. AGRONET. 2018. Reporte: Área, Producción y Rendimiento Nacional por Cultivo. Disponible desde Internet en: https://www.agronet.gov.co/estadistica/Paginas/home. aspx?cod=1\# (con acceso el 05/05/2020) 
2. AIME, M.C.; ABBASI, M. 2018. Puccinia modiolae in North America: Distribution and natural host range. MycoKeys. 39:63-73. https://doi.org/10.3897/mycokeys.39.27378

3. ALBU, S.; BLOMQUIST, C.L.; SCHECK, H.J.; DOYLE, V.P. 2019. First report of rust on Modiola caroliniana caused by Puccinia modiolae in California. Plant Disease. 103(11):2961. https://doi.org/10.1094/pdis-02-19-0303-pdn

4. ÁVILA, C.; ROBLES, A.; PINZÓN, S.; MIRANDA, D.; FISCHER, G. 2013. Tecnologías locales para los sistemas de producción de frutales caducifolios en zonas productoras de Colombia y sus limitantes tecnológicas. En: Miranda, D.; Fischer, G.; Carranza, C. (eds). Los frutales caducifolios en Colombia. Situación actual, sistemas de cultivo y plan de desarrollo. Equilibrio Gráfico Editorial Ltda (Bogotá D.C.) p.115-149.

5. CALHA, I.M.; SOUSA, E.; GONZALEZ-ANDUJAR, J.L. 2014. Infestation maps and spatial stability of main weed species in maize culture. Planta daninha. 32(2):275-282. https://doi.org/10.1590/S0100-83582014000200004

6. CAMPO, A.; DUVAL, V. 2014. Diversidad y valor de importancia para la conservación de la vegetación natural. Parque nacional Lihué Calele (Argentina). Anales de Geografía. 34(2):25-42.

https://doi.org/10.5209/rev_AGUC.2014.v34.n2.47071

7. CARDENAL, Z.C.; TORRES, D.F.; DOTOR, M.Y.; MORILLO, A.C. 2016. Caracterización del banco activo de semillas en cultivos de zanahoria del municipio de Villa Pinzón (Cundinamarca). Rev U.D.C.A Act. \& Div. Cient. 19(2):297-306

https://doi.org/10.31910/rudca.v19.n2.2016.83

8. CASTRO, V.; ALVARADO, L.; TEJADA, J.; BORJAS, R.; BELLO, S.; DA COSTA, P.; HELGOTT, S.; JULCA, A. 2019. Manual de malezas asociadas al cultivo de Café en la selva central del Perú. Universidad Nacional Agraria La Molina (Perú). 43p

9. COBB, A.; READE, J. 2010. Herbicides and plant physiology 2nd ed. John Wiley and Son (Oxford). 296p.

10. CONCENÇO, G.; ANDRES, A.; SCHREIBER, F.; SILVA, A.F.; MOISINHO, I.S.; CORADINI, M.C. 2019. Characterization of the weed flora in rice areas under distinct cropping systems and herbicide managements. Planta Daninha. 37:e019193419. https://doi.org/10.1590/S0100-83582019370100089

11. DEVORE, J. 2012. Probabilidad y estadística para ingeniería y ciencias 8va ed. Cengage Learning Editores S.A. (México D.F.). 63p.
12. DUKE, S.O. 2018. Weed physiology. CRC Press (New York). 176p.

13. FACHINELLO, J.C.; PASA, M.; SCHMITZ, J.; BETEMPS, D.L. 2011. Situação e perspectivas da fruticultura de clima temperado no Brasil. Rev. Bras. Frutic. 33( ${ }^{\circ}$ especial):109-120. http://dx.doi.org/10.1590/S0100-29452011000500014

14. FUENTES, C.; ERASO, E.R.; SEQUEDA, O.A.; PIEDRAHITA, W. 2011. Flora arvense del altiplano Cundiboyacense de Colombia. Universidad Nacional de Colombia (Bogotá). 348p.

15. FUENTES, C.L.; OSORIO, A.S.; GRANADOS, J.C.; PIEDRAHITA, W. 2006. Flora arvense asociada con el cultivo del arroz en el departamento del Tolima-Colombia. Bayer Cropscience. Universidad Nacional de Colombia (Bogotá). 256p.

16. GÁMEZ, A.; ROJAS, L.; JARAMILLO, M.; CRUZ, E.; HOYOS, V.; CEPEDA, J.; PLAZA, G.A. 2018. Guía Ilustrada De Plantas Arvenses Del Centro Agropecuario Marengo (CAM). Universidad Nacional de Colombia (Bogotá). 382p.

17. GONZALES-OREJA, J.; DÍAZ, A.; HERNÁNDEZ, L.; BUZO, D.; BONACHE, C. 2010. Evaluación de estimadores no paramétricos de la riqueza de especies. Un ejemplo con aves en áreas verdes de la ciudad de Puebla, México. Animal Biodiversity and Conservation. 33(1):3145.

18. HOYOS, V.; MARTÍNEZ, M.; PLAZA, G. 2015. Malezas asociadas a los cultivos de cítricos, guayaba, maracuyá y piña en el departamento del Meta, Colombia. Rev. Col. Ciencias Hortícolas. 9(2):247-258.

http://dx.doi.org/10.17584/rcch.2015v9i2.4181

19. IDEAM. 2012. Características climatológicas de ciudades principales y municipios turísticos. Disponible en internet desde:

http://www.ideam.gov.co/documents/21021/21789/ 1Sitios + turisticos2.pdf/cd4106e9-d608-4c29-91cc16bee9151ddd (con acceso el 12/08/2019).

20. JABRAN, K.; CHAUHAN, B. 2018. Non-chemical Weed Control. ${ }^{\text {st }}$ ed. Academic press (USA). 172p.

21. JAMAICA, D.; PLAZA, G. 2014. Evaluation of various conventional methods for sampling weeds in potato and spinach crops. Agr. Col. 32(1):36-43. http://dx.doi.org/10.15446/agron.colomb.v32n1.39613

22. JUÁREZ, A.; NATIVIDAD, H.; MARTÍNEZ, J.; UMAÑA, M. 2007. Diversidad y estructura de la selva mediana 
subperennifolia de Acapulco, Gro., México. Rev. Iberoamericana de las Ciencias Biológicas y Agropecuarias. $5(10): 1-20$.

23. MAGURRAN, A. 2004. Measuring Biological Diversity. $1^{\text {st }}$ ed. Blackwell Science LTD (Australia). 256p.

24. MARQUEZ, T. 2019. Gestión integrada de malas hierbas en frutales de pepita y de hueso. Rev. Fruticultura. 67:54-65.

25. NAGENDRA, H. 2002. Opposite trends in response for the Shannon and Simpson indices of landscape diversity. Applied Geography. 22:175-186. https://doi.org/10.1016/S0143-6228(02)00002-4

26. PATIÑO, L.; MIRANDA, D. 2013. Situación actual de los frutales caducifolios en el mundo y en Colombia. En: Miranda, D.; Fischer, G.; Carranza, C. (eds). Los frutales caducifolios en Colombia. Situación actual, sistemas de cultivo y plan de desarrollo. Equilibrio Gráfico Editorial Ltda (Bogotá D.C.) p.9-20.

27. PINZÓN, E.; MORILLO, A.C.; FISCHER, G. 2014. Aspectos fisiológicos del duraznero (Prunus persica [L.] Batsch) en el trópico alto. Una revisión. Rev. U.D.C.A Act. \& Div. Cient. 17(2):401-411.

https://doi.org/10.31910/rudca.v17.n2.2014.243

28. PLAZA, G.A.; PEDRAZA, M. 2007. Reconocimiento y caracterización ecológica de la flora arvense asociada al cultivo de uchuva. Agr. Col. 25(2):306-313.

29. PRATES, C.J.N.; VIANA, A.E.S.; CARDOSO, A.D.; SÃO JOSÉ, A.R.; VIANA, B.A.R.; DUTRA, F.V. 2019. Weed phytosociology in cassava cultivation in two periods in southwestern Bahia, Brazil. Planta Daninha. 37:e019208668. https://doi.org/10.1590/S0100-83582019370100107

30. QUINTERO-PERTUZ, I.; CARBONÓ-DELAHOZ, E.; JARMA-OROZCO, A. 2020. Weeds associated with banana crops in Magdalena department, Colombia. Planta Daninha. 38:e020217466. https://doi.org/10.1590/S0100-83582020380100015
31. RAMÍREZ, J.; HOYOS, V.; PLAZA, G. 2015. Phytosociology of weeds associated with rice crops in the department of Tolima, Colombia. Agr. Col. 33(1):64-73. https://doi.org/10.15446/agron.colomb.v33n1.46747

32. RODRÍGUEZ, J. 2013. Ecología. 3er ed. Ediciones Pirámide (Madrid). 504p.

33. RODRÍGUEZ, M.; PLAZA, G.; GIL, R.; CHAVES, B.; JIMÉNEZ, J. 2008. Reconocimiento y fluctuación poblacional arvense en el cultivo de espinaca (Spinacea oleracea L.) para el municipio de Cota, Cundinamarca. Agr. Col. 26(1):87-96.

34. SOMERVILLE, G.J.; SØNDERSKOV, M.; MATHIASSEN, S.K.; METCALFE, H. 2020. Spatial Modelling of WithinField Weed Populations; a Review. Agronomy. 10:1044. https://doi.org/10.3390/agronomy10071044

35. STORKEY, J.; NEVE, P. 2018. What good is weed diversity? Weed Research. 58:239-43. https://doi.org/10.1111/wre.12310

36. TAUSEEF, M.; IHSAN, F; NAZIR, W.; FAROOQ, J. 2012. Weed flora and importance value index (IVI) of the weeds in cotton crop fields in the region of Khanewal, Pakistan. J. Weed Science Res. 18(3):319-330.

37. TIBUHWA, D. 2016. Oxalis corniculata L. in Tanzania: traditional use, cytotoxicity and antimicrobial activities. J. Applied Biosciences. 105(1):10055. https://doi.org/10.4314/jab.v105i1.2

38. VERA, A.; PALACIOS, Z.; LIUBA, S.; MENDOZA, H. 2018. Diversidad y análisis fitosociológico de malezas en un cultivo de musáceas del trópico ecuatoriano. Agriscientia. 35(2):43-52. https://doi.org/10.31047/1668.298x.v35.n2.22966

39. ZIMDAHL, R.L. 2018. Fundamentals of weed science. $5^{\text {th }}$ ed. Academic Press (USA). 758p. 